\title{
Systema reticulo-endothelial e hemo- lymphopoiese, com noções de outras propriedades funccionaes do systema.
}

\author{
Pelo Dr. J. Oria \\ Lição do Laboratorio de Histologia e \\ Embryologia da Fac. de Med. de S. Paulo
}

\section{HISTOGENESE DO SYSTEMA}

O mesenchyma derivam não só os diversos elementos do connectivo: as cellulas com as diversas formas de substancia fundamental, como ainda os componentes do sangue e da lympha.

A cellula mesenchymal não evolue em todos os pontos do organismo: permanece indifferente em sectores especiaes, de tal modo que está prompta a dar em qualquer momento, tanto elementos histioides, como componentes hematicos. Esta cellula assim indifferente e polyvalente chamase "hemohistioblasto" (FERrata) ou "histiocyto"

\section{ESCHEMA DO S. R. E.}

Para se reconhecer quaes os representantes desse elemento cellular polyvalente, procurou-se reunil-os num eschema.

Baseando-se no methodo de Goldmann das injecções intravitam verificou-se que assumiam de preferencia as côres certos representantes daquella que occupavam no organismo postos e adaptaçôes variaveis. Prescindindo dos endothelios communs dos vasos e dos fibroblastos que já são elementos mais differenciados e fixos e que, se assumem as côres, o fazem menos intensamente (endothelios) ou de um modo differente (nos fibroblastos, o corante penetrando toma uma disposição bacilliforme), sabemos depois de Aschoff que são chromophilas: 
a) as cellulas de reticulo da polpa branca esplenica, dos nodulos certicaes e cordóes medullares dos ganglios lymphaticos, dos centros germinativos dos folliculos lymphoides, etc..

b) os reticulo-endothelios (armazenando as côres mais facil e intensamente que as primeiras), dos seios lymphaticos, dos seios venosos do baço, dos capillares do figado, medulla ossea, cortex suprarenal e hypophyse.

c) os elementos moveis em repouso do connectivo frouxo diffuso das serosas, das mucosas e sub-mucosas, do derma e hypoderma, da adventicia dos vasos e de qualquer forma de tecido connectivo intersticial dos orgãos. Esses elementos que Aschoff-Kryono denominam de histiocytos typicos, já anteriormente tinham sido por varios A.A. reconhecidos nas suas morphologia e propriedades. Os que estão no frouxó das serosas tinham sido vistos por RaNVIER (1890) que os denominou de "clasmatocytos", os da adventicia dos vasos correspondem ás "cellulas adventiciaes de MarGhand".

São ainda chromophilas: as cellulas adiposas, as cellulas lipoidicas de Ciaccio, modalidades funccionaes de histiocytos communs. Ha duvidas sobre a chromophilia das cellulas pígmentares.

\section{PROPRIEDADES GERAES DAS CELLULAS DO SYSTEMA}

Os grupos $a-b-c$ estão ligados por uma propriedade fundamental: são elementos não differenciados do connectivo que assumem vitalmente as particulas corantes. Assumem além do mais, particulas de metaes colloidaes ou qualquer outra substancia em suspensão introduzida no organismo.

Esta propriedade que muito provavelmente se exerce nelles por um mechanismo de phagocytose, faz-nos deduzir a existencia duma actividade fundamental. Quero dizer que as cellulas $a-b-c$ possuem no organismo uma actividade de defeza podendo exercel-a in loco, como tambem se mobilizando para isso; tal actividade, nos explica como podem ser englobados as particulas extranhas, os globulos vermelhos e brancos velhos, os germens, etc. etc..

Este facto que fôra já visto por KuPFER (1876) nas cellulas estrelladas dos capillares hepaticos (phagocytose de 
residuos dos globulos vermelhos), ficou definitivamente assentado por Metchnikoff, que definiu o valor das cellulas mesenchymaes na defeza organica.

\section{ESTROMA DO SYSTEMA}

Mas ha ainda um dispositivo anatomico e histogenetico que liga entre si aquelles tres grupos: as cellulas estão dispostas sempre em contiguidade do tecido reticular, tecido esse que é elaborado por ellas mesmas. Foi assim que Aschoff estabeleceu o conceito de "systema reticulo-endothelial", que abrange em sentido estricto os tres grupos cellulares com seu estroma reticulado.

\section{CONCEITO DE VOLTERPA}

Na denominação "reticulo endothelial", AsGHoFf soffreu a critica de que a expressão não refere os nomes dos tres grupos do systema. Neste caso, si quizermos com VolTERRA, chamar de histiocytos de um modo geral, tanto os histiocytos typicos do connectivo frouxo como as cellulas reticulares com ou não disposição endothelial, chegaremos ao conceito de systema reticulo-histiocytario. Comtudo a denominação de "systema reticulo-endothelial" ou abreviadamente S. R. E., foi consagrada pelo uso e é a que conservaremos d'ora avante.

\section{CONCLUSÃO PRELIMINAR}

O S. R. E. consta de uma parte cellular, histiocytaria, que comprehende os reticulocytos, os reticulo-endothelios e os histiocytos typicos, e que se evidencia pelo methodo das injecções vitaes; de uma parte estromatica, que comprehende um reticulo, lamellar ou não, que se denuncia pela impregnação argento-ammoniacal.

O S. R. E. se distribue em todos os pontos do organismo em que houver capillares, mas, em certos orgãos elle é mais abundante e ahi goza de funcções especiaes. Essas funcções são tão importantes, que para seu conhecimento moveram um exercito de pesquizadores no terreno das sciencias experimentaes. Embora ainda não bem esclarecida, 
pode-se dizer que é enorme a contribuição dâda a respeito pelos A.A. O S. R. E. mobilizou não só a Morphologia, como a Physiologia, a Pathologia Geral, a Microbiologia, a Pathologia Especial.

Aschoff, levando em conta suas funcções certas ou provaveis ou possiveis, chama-o de apparelho do metabolismo material, dando-lhe a dignidade de systema ao lado dos outros quatro systemas de correlação: nervoso, chimico, organico e psychico.

"Actualmente, - diz Pianese, este quinto systema ameaca assumir importancia ainda maior dos outros quatro, pois é certo que hoje elle domina em biologia, especialmente nos sectores mais tormentosos e atormentados desta: a hemolymphopoiese, a biligenese, a immunidade, o metabolismo intermediario, as secreções internas..

\section{DISTRIBUIĢÃO DO S. R. E. PELOS ORG̃̃OS PRINCIPAES}

1..$^{\circ}$ Figado: nos capillares intralobulares hepaticos o forro endothelial das cellulas estrelladas de KupFER é seu representante. São elementos que possuem prolongamentos anastomoticos entre si e estão contiguos ao reticulo que elles mesmos elaboraram; gozam das propriedades geraes do systema, como por exemplo a hematopoiese (hematopoiese intravascular do embryão).

No figado ainda ha elementos histiocytarios que não estão em posição endothelial: occupam o estroma do conjunctivo intersticial aos lobulos e a adventicia dos vasos dessa região. Esses histiocytos intersticiaes têm no feto uma actividade hematopoietica toda especial (hematopoiese extravascular).

As duas maneiras diversas de formação do sangue no figado, serão estudadas mais adiante.

\section{2..$^{\circ}$ Orgãos hematopoieticos:}

a) $\mathrm{Na}$ medulla ossea tanto as cellulas reticulares do estroma, como os reticulo-endothelio dos capillares têm funcção hematopoietica que se realiza na vida fetal com. grande intensidade e que persiste residualmente no adulto.

Do histiocyto da medulla ossea deriva uma cellula que Ferrata chama hemocytoblasto: desta cellula em diante, 
só poderemos ter elementos hematicos erythrocytarios e granulocytarios. Disso ainda falaremos detalhadamente.

b) No baço, tanto as cellulas reticulares do estroma da polpa branca (folliculos de Malpighi e cordões de Bilroth), como os reticulo-endothelios dos seios venosos, entram a fazer parte do systema. Esses elementos durante a vida fetal gozam de propriedade erythroblastica como os da medulla ossea, mas logo depois do nascimento abandonam-na para gozar de outras propriedades (formação de lymphocytos, p.ex.).

As cellulas reticulares do baço estão presas a um reticulo que forma fino arcabouço; o reticulo dos endothelios dos seios venosos se dispõem em anneis em torno da luz dos seios (fibras annullares de Henle). As cellulas endotheliaes muito alongadas, estão cimentadas nesse reticulo annular de tal modo, a compôrem aquella figura classica do reticulo fenestrado com um endothelio discontinuo.

Os histiocytos fixos do baço perdem frequentemente connexão com o reticulo; mobilizam-se e tornam-se livres, para melhor exercerem sua actividade phagocytaria (de globulos vermelhos, de pigmento ferrico, por exemplo); podem mesmo uma vez destacados, cahirem no sangue circulante, como foi visto experimentalmente ou em certos casos pathologicos. Esses histiocytos livres têm o mesmo aspecto e valor funccional dos monocytos.

c) Nos ganglios lymphaticos, a mesma arquitectura do baço: os representantes do systema são ainda as cellulas reticulares da polpa lymphoide e os endothelios reticulares lymphaticos. A funcção lymphopoietica (formação de lymphocytos) é ainda exercida por esse sector do S. R. E. Por outro lado, os ganglios lymphaticos em muitos casos experimentaes ou pathologicos, podem adquirir certas propriedades do baço, como por exemplo, a phagocytose dos globulos vermelhos e a elaboração da molecula de homoglobina. Os histiocytos do ganglio podem ainda se destacar e cahir no sangue. Commummente se encontram histiocytos livres na lympha.

3.) Os histiocytos diffusos pelo connectivo intersticial das mucosas e sub-mucosas, do derma e hypoderma, de certas glandulas, etc.; os endothelios da cortex e da capsula suprarenal, do lobo anterior da hypophyse, etc., pertencem ao S. R. E.. Esses histiocytos gozam da propriedade latente de formar cellulas do sangue, que desperta sob estimulos 
anormaes: formam por isso o sector hematopoietico diffuso de Ferrata. Frequentemente dão origem a cellulas do connectivo já conhecidas: plasmocytos, lymphocytos histioides, endothelios vasculares communs, fibroblastos e suas variedades, assim como a substancia fundamental de natureza collagena e elastica. No derma o S. R. E. é ainda representado pelas cellulas pigmentares, cuja funcção melanogenetica é de alta importancia.

\section{FUNCÇôES DO S. R. E.}

Podemos fazer o seguinte quadro das funcções mais importantes do S. R. E.:

1-Funcção hemolymphopoietica.

2 - $"$ hemocatheretica e sidérotica (metabolismo do

3 - " biligenetica.

ferro).

4 - Outras funcêões metabolicas $\left\{\begin{array}{c}\text { dos lipoides e gorduras, } \\ \text { dos proteicos. }\end{array}\right.$

5 - S. R. E. e defeza cellular, immunidade, etc.

1) FUNGÇÃo HEMOLYMPHOPOIETICA - O histiocyto ou hemohistioblasto, que como vimos se encontra diffuso em todo o connectivo, encerra no adulto o poder latente de dar origem ás cellulas do sangue e da lympha. Porém, como não ha exigencia no organismo adulto normal, de a todo o momento tal elemento generico nos fornecer componentes lymphó-hematicos, a hemolymphopoiese desde a vida fetal, fica limitada a orgãos especiaes. De limitada só virá a ser diffusa, quando houver demanda funccional exigida por estimulos abnormes; assim se comprehenderá como em casos pathologicos despertam os histiocytos de todos os sectores do systema.

THORIAS PARA EXPLICAR A GENESE DO SANGUE. CONCEITO DE HEMOCYTOBLASTO

Nos orgãos ditos hematopeieticos, o hemohistioblasto durante a vida intra-uterina produz um primeiro elemento fundamental hemo-polyvalente: o hemocytoblasto. Tal cellula daqui em diante, terá por si só e exclusivamente, aptidão de gerar os componentes erythrocytarios, granulocytarios e lymphocytarios (eschema unicista de Ferrata). Se- 
gundo este eschema, tambem os monocytos se originariam daquella cellula mater.

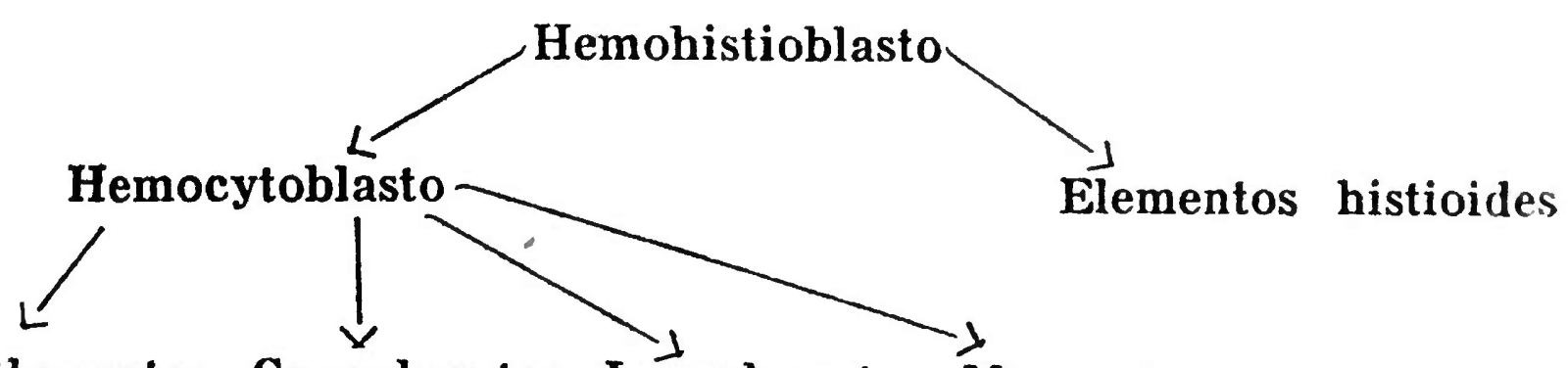

Erythrocytos Granulocytos Lymphocytos Monocytos

Os partidarios desta theoria monophylectica sustentam que a cellula sanguinea ancestral commum persiste durante a vida post-fetal, na medulla ossea, baço e ganglios, podendo apperecer no sangue circulante em varias condições pathologicas. Assim persistente, se explica como, o hemocytoblasto que normalmente nos ganglios só fornece lymphocytos, pode anormalmente dar erythrocytos e granulocytos.

Porém, ha A. A. que não admittem a persistencia durante a vida extra-uterina da cellula polyvalente daquelle typo. Para estes, o antepassado cellular commum desapparece antes do nascimento, logo apoz ter-se differenciado em duas especies cellulares independentes entre si: uma myeloide, porque existente na medulla ossea, apta a gerar granulocytos e hemacias, outra lymphoide, propria do baço e dos ganglios, especializada a dar somente lymphocytos (theoria dualista de Naegeli).

Segundo a escola de Aschoff, os monocytos por sua vez, derivariam directamente dos histiocytos sem o intermedio do hemocytoblasto.

A concepção de Ferrata com esta ligeira modificação de Aschoff, parece-nos a mais acertada. $O$ hemocytoblasto persiste e dá elementos myeloides e lymphoides, sem o que não se explicaria o facto de num ganglio ser possivel a formação de globulos vermelhos e granulocytos.

Podia-se objectar nesse caso, que tanto os lymphocytos (normaes do ganglio) como os granulocytos e erythrocytos (anormaes), se originassem já não do hemocytoblasto, mas da cellula mais antiga cuja persistencia ficou comprovada: o hemohistioblasto. Desse modo passaremos a uma theoria polyphyletica

Como se pode deduzir do eschema acima, ha dois grupos especificos de tecidos hematopoieticos, um myeloide (myeloblastico e erythroblastico), outro lymphoide (lym- 
phoblastico), e um grupo generico, que é o proprio S. R. E. fornecendo directamente os monocytos do sangue. Não intervém o hemocytoblasto.

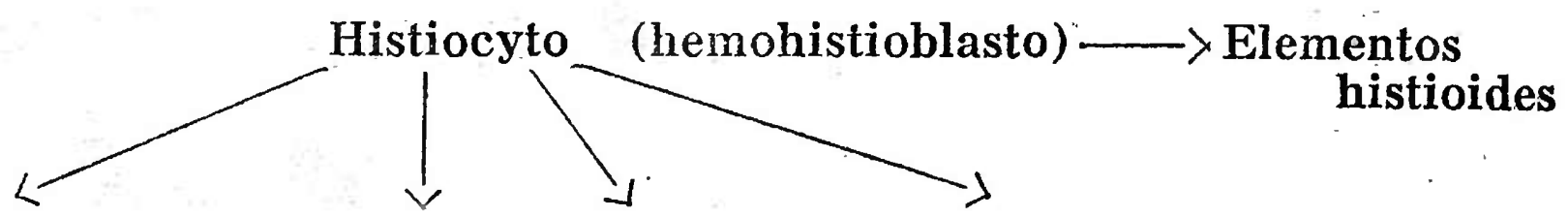

Myeloblasto Erythroblasto Lymphoblasto Monocyto

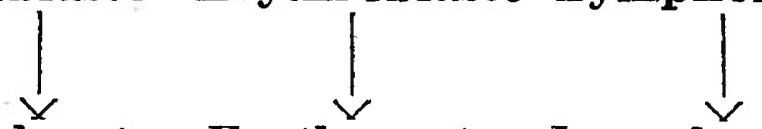

Granulocyto Erythrocyto Lymphocyto.

Entretanto, não podemos afastar o conceito de hemocytoblasto: esta cellula existe e foi demonstrada morphologicamente pela escola italiana. A unica duvida que esta escola não esclareceu ainda, refere-se a origem dos monocytos.

\section{PHASES DA HISTOGENESE DO SANGUE}

Discutido o conceito do hemocytoblasto, vejamos em que phases da vida intra-uterina se realiza a histogenese do sangue.

1. ${ }^{a}$ - Phase embryonal ou diffusa.

$2 .^{a}$ - Phase hepatica.

3. ${ }^{\mathrm{a}}$ - Phase fetal.

1.a) - Phase embryonal ou diffusa: nesta primeira phase, a formação do sangue é dita diffusa porque no embryão não se formaram ainda orgãos especificos hemopoieticos. A area embryonaria composta de tres folhetos fundamentaes se continua lateralmente com uma area extraembryonaria. Esta area extra-embryonaria está tambem formada por tres folhetos fundamentaes: ectoderma, endoderma e mesoderma. Estamos nas primeiras semanas de vida: na parede desta area, vêmos apparecer ilhotas de elementos cellulares derivados do mesoderma (ilhotas sanguineas de Wolff). Estas, examinadas ao microscopio, apparecem como brótos cellulares, dentro ou não dos primeiros capillares. Os primeiros capillares formam-se á custa da canalização da substancia fundamental e são revestidos pelos elementos mesodermicos dispostos pela primeira vez em en- 
dothelio. Esse endothelio dá origem ás cellulas que cahem na luz e ficam livres no interior dos capillares: são hemocytoblastos embryonarios. Os elementos endotheliaes correspondem a hemohistioblastos. A hematopoiese desse modo é dita intra-vascular (Maximov). Mas as cellulas mesodermicas independentemente de uma adaptação endothelial, podem dar origem a hemocytoblastos, neste caso a hematopoiese é dita extra-vascular.

Do hemocytoblasto embryonal originam-se somente globulos vermelhos embryonarios, globulos grandes em relação aos do adulto normal e por isso mesmo chamados "megalocytos" Não ha granulocytos e lymphocytos nesta phase. Para chegar a megalocyto o hemocytoblasto embryonal passa pelas seguintes phases (FERRATA):

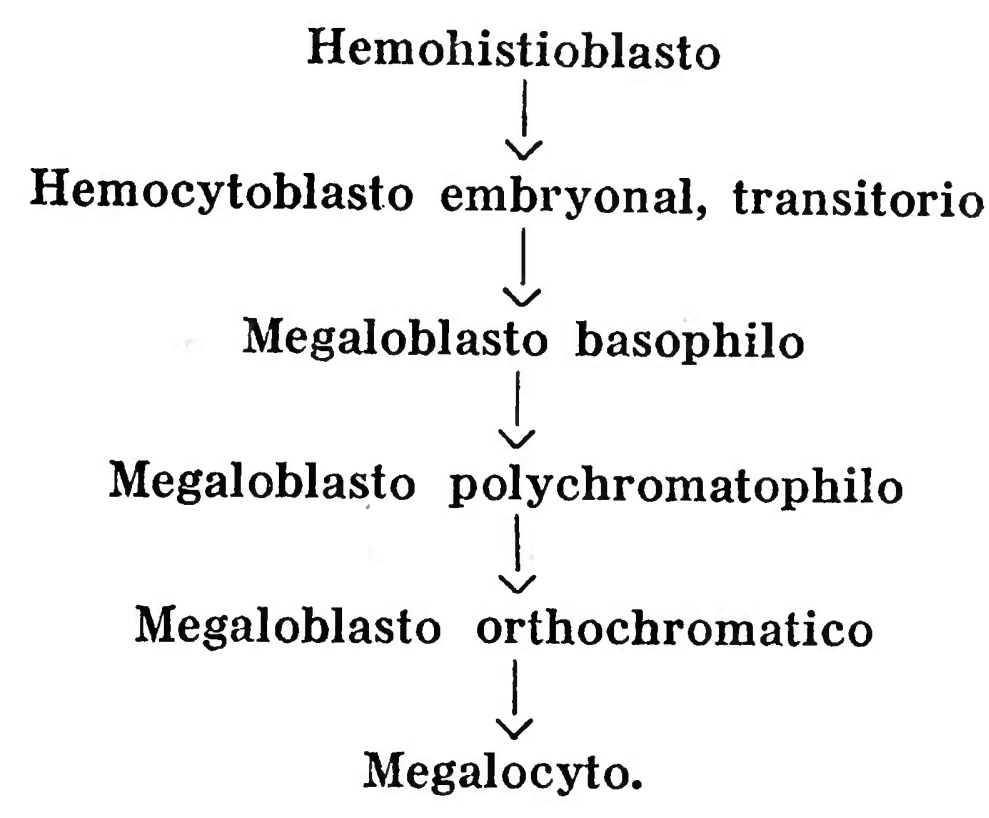

Estudaremos praticamente cada um desses elementos e o mechanismo gradual de sua maturação.

2.a - Phase hepatica: aqui a hematopoiese se localiza no figado. Estamos pelas 10.a $150^{a}$ semanas. Os elementos glandulares hepaticos acham-se dispostos em trabeculas, entre as quaes se encontram os capillares sanguineos revestidos das cellulas de KUPFER. Onde não existem capillares e cellulas hepaticas, o mesenchyma penetrára com seus elementos cellulares.

Nesta phase temos ainda um residuo da hematopoiese diffusa: nos capillares hepaticos as cellulas de KUPFER, embora transitoriamente dão origem a megaloblastos. 
Concomitantemente, fóra dos capillares, observamos o primeiro esboço do segundo typo de formação do sangue: no tecido mesenchymal intersticial, apparecem os primeiros globulos vermelhos normaes (os correspondentes aos do adulto) e os primeiros leucocytos granulosos. Esta erythro-e granulopoiese hepatica dura poucas semanas, porque lá pelo 4..$^{\circ} \mathrm{mez}$, vamos assistil-a no baço e, sobretudo na medulla ossea que resultará então o sector hematopoietico mais importante do systema.

Resumindo: durante o $3 .^{\circ} \mathrm{mez}$, ha no figado uma hematopoiese intra-capillar em que o reticulo endothelio dá formação de globulos vermelhos embryonarios (megalocytos) e outra extra-capillar, em que os histiocytos do connectivo intersticial produzem elementos das series erythro e granulocytarias.

Aquella primeira forma de hematopoiese hepatica, intra-vascular ou megaloblastica, desapparece rapidamente; no adulto, entretanto, pode apparecer em certos casos pathologicos (anemia perniciosa).

A segunda forma, extra-vascular, é identica á que vamos estudar na 3.a phase.

3.a) - Phase fetal: a hematopoiese definitiva (formação de erythrocytos, leucocytos e megacaryocytos) se assesta na medulla de todos os ossos durante toda a vida fetal, persistindo no adulto, nos ossos curtos e epiphyses dos ossos longos.

O baço fetal, até á epoca do nascimento, produz tambem os mesmos elementos da medulla ossea. Mas, tanto ao baço como aos ganglios, caberá o papel de producção dos lymphocytos do sangue e da lympha. Podemos verificar uma reproducção de hematopoiese fetal em adultos: nos casos de anemia e nos casos em que os globulos brancos são solicitados mais abundantemente em circulo. Nesses casos então, para compensar o deficit sanguineo, não só se tem demanda funccional da medulla ossea com hyperplasia da mesma, como ainda do proprio baço e dos ganglios, os quaes dão origem á formas erytho - e granulocytarias e a megacaryocytos.

O eschema de Ferrata demonstra claramente o caso normal fetal e a sua reproducção anormal. 


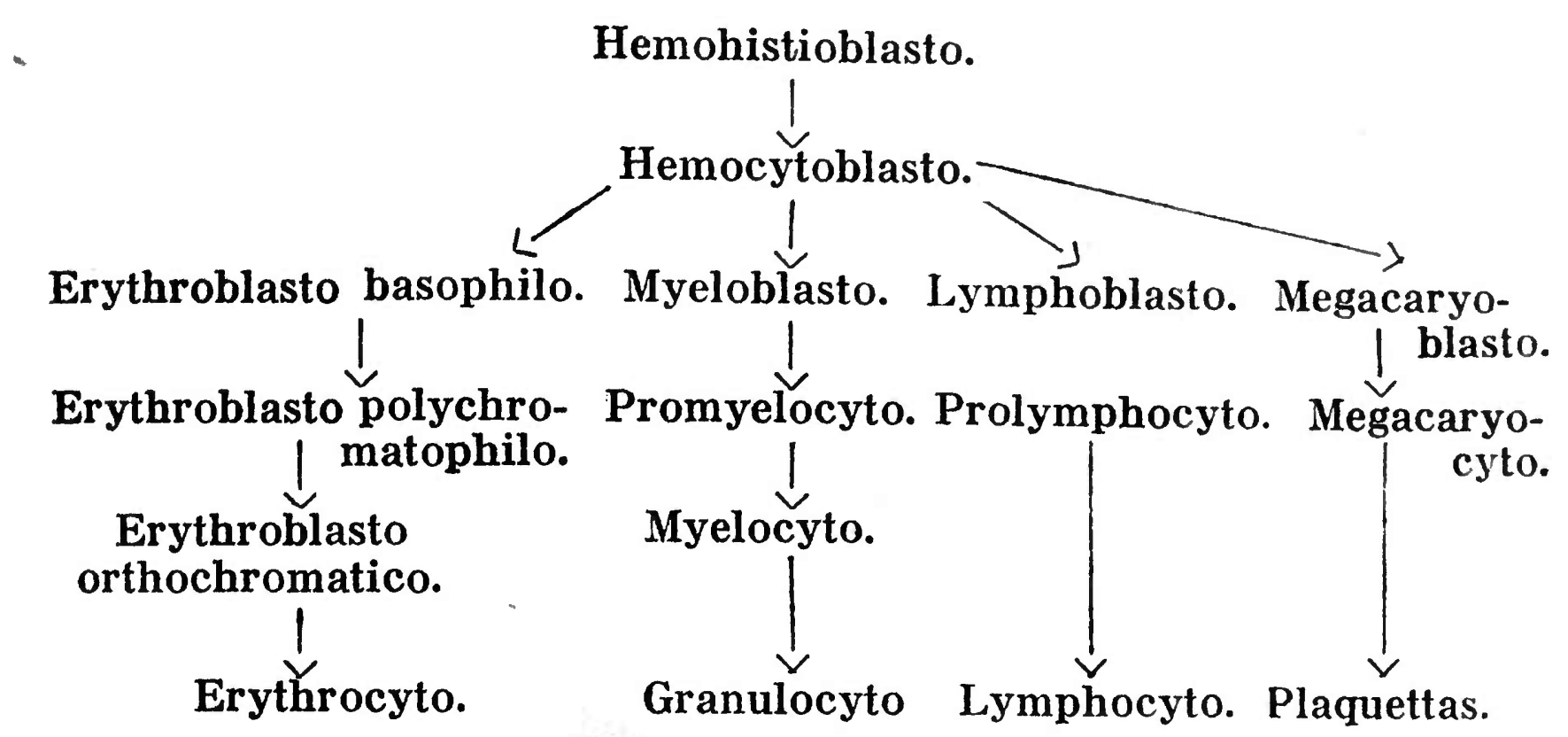

Limito-me a acennar a escala: em estudo pratico estudaremos os caracteres differenciaes desses elementos.

Quanto aos monocytos: de accordo com Ferrata, formar-se-iam na medulla ossea derivando do monoblasto, que por sua vez derivasse do hemocytoblasto. E' conveniente admittir, porém, com Aschoff que o monocyto se origine directamente não só do histiocyto da medulla ossea, como do baço, dos ganglios e de qualquer outro sector do S. R. E.

2) - FUnção hemocatheretica E Hemosiderotica - Reconhecida no S. R. E. a actividade formadora de sangue, vamos estudar agora sua acção hemo-destruidora. Os globulos vermelhos e brancos velhos ou seus residuos são destruidos ao nivel do baço por um mecanismo de phagocytose. Nos casos anormaes, outros sectores do S. R. E. podem adquirir essa funcção, como por exemplo, os do ganglio lymphatico.

As cellulas reticulares, os reticulo-endothelios, esses mesmos elementos destacados de seu reticulo, numa palavra os histiocytos esplenicos fixos e livres, gozam da propriedade de englobar globulos vermelhos inteiros ou seus fragmentos. Estes, uma vez phagocytados, são destruidos, não sem que o proprio S. R. E., deixe de utilizar os productos dessa destruição: dahi decorre que o pigmento hemoglobinico é elaborado e transformado em pigmento ferrico e pigmento não ferrico. O pigmento ferrico (hemosiderina) que é reconhecido facilmente pelo seu aspecto e pelas propriedades chimicas (dá reacção do ferro), é aproveitado muito provavelmente no metabolismo: servirá de material para globulos vermelhos novos. Além desse ferro endogeno, o S. R. 
E. tem ainda a propriedade de armazenar o ferro alimentar e medicamentoso.

O pigmento não ferrico (hematoidina), que é isomero da bilirubina, darą origem ao pigmento biliar.

3) - FUnção BILIGENICA - Que o pigmento biliar derive do pigmento hematico não ferrico, sabe-se desde Wirchov: nos fócos hemorrhagicos se encontram crystaes da substancia identica á bilirubina. Da mesma maneira "in vitro", partindo, da hemoglobina pode-se chegar muito proximo a bilirubina. Esses factos fizeram suppor que a bile pudesse ser fabricada fóra do figado, isto é, fóra da sua cellula glandular.

Muitos A.A. portanto, procuraram distinguir uma biligenese extra-hepatica, que corresse por conta do S. R. E. Outros, sustentaram a ideia antiga de que sem o figado não se pode fabricar pigmento biliar. Entre o debate das "theorias anhepatocellular e hepatocellular" ha ainda muita incerteza, quer no terreno experimental, quer no clinico.

Em 1886, Staldemann e Stern, experimentaram gansos envenenados com hydrogenio arsenical, substancia fortemente hemolytica; em seguida de alguns animaes extirparam o figado e no fim de algum tempo verificavam nesses uma simples eliminação da hemoglobina (hemoglobinuria). Nos gansos intactos viam surgir a ictericia: isto é, a bile era fabricada em tal quantidade no figado, que não podia escoar-se inteiramente pelas vias naturaes, passando em circulo. Minkowski repetiu essas experiencias; o mesmo resultado: "não ha ictericia sem figado"

No entretanto, MÁC NEE, ainda em gansos, tornou a repetil-as: apezar de extirpar o figado sem deixar traços, verificava sempre leve producção de pigmentos biliares.

Como explicar esses factos apparentemente contradictorios?

$E^{\prime}$ que nos gansos sem figado, retira-se quasi todo o S. R. E., que nas aves se localiza de preferencia naquelle orgão, restando somente o S. R. E. esplenico, mais reduzido. Dahi, a quasi não producção de bile quando desses animaes se tira o figado com as suas cellulas de KuPFER; a pouca que se produz é originada dos histiocytos do baço.

Mais tarde, fazendo experiencias em Mammiferos, outros A.A. usaram do seguinte methodo: com a injecção 
de substancias colloidaes que se armazenassem em todos os pontos do S. R. E. pretendiam primeiramente sobrecarregar as cellulas do systema, de tal modo a emperrar suas funcções (inclusive a biligenetica): é o que se chama "bloqueio"

Alguns A.A. bloqueiaram o S. R. E. de ratos com collargol, extirpando contemporaneamente o baço; ao praticarem a seguir a hemolyse experimental não deveriam naturalmente observar a ictericia. Todavia, mesmo assim o pigmento biliar se forma. Para salvar a situação desse caso, admitte-se que o bloqeio é um methodo experimental insufficiente; com elle as cellulas do systema nunca são inteiramente obstadas nas suas funcções.

Pearce, sem se utilizar do bloqueio conseguiu demonstrar o valor biligenetico do S. R. E. esplenico: este A., injectando soro hemolytico em cães com e sem baço, viu que nos inteiros apparece ictericia, nos sacrificados, não.

Whipple e Hooper, usando de um meio pratico de se eliminar o figado sem extirpal-o (fistula de Eck: ligadúra da arteria hepatica e anastomose da veia porta com as veias super-hepaticas) ainda observaram a ictericia em animaes experimentados.

Mann, Magatta, Bolkmann, anastomosando as veias do figado e em seguida extirpando esse orgão, sempre obtiveram com dosagem meticulosa, a bilirubina no sangue.

Aschoff controlou e approvou esses resultados de tal modo que hoje pode-se concluir o seguinte: parece certo que cabe ao S. R. E. manifesto papel na biligenese; esta, normalmente e em Mammiferos, se realiza sobretudo no baço, e em casos anormaes nos ganglios, etc. e mesmo no sangue circulante; o figado, si tem um papel biligenetico, cabe-o ás cellulas de KupFER; quantos ás cellulas glandulares hepaticas só armazenariam e veiculariam a bile formada no S. R. E. esplenico; o pigmento formado no baço chega ao figado pela veia esplenica e nella pode ser dosado.

Outros dados comprovam esse modo de pensar, sobretudo os de ordem clinica: na ictericia hemolytica, o baço destroe globulos vermelhos em grande quantidade e dahi decorre a hyper-producção biliar; na ictericia catarrhal, a cellula hepatica soffre processos degenerativos e entra em 
hypo-funcção; neste caso seria razoavel que se formasse menor quantidade de bile; entretanto, ella continua se produzindo, porém, fóra da cellula hepatica; não se escoa pelas vias excrectoras como normalmente, porque a glandula com sua funcção alterada deixa-a passar para o sangue.

4) - metabolismo dos lipoides e dos proteicos - Temse observado experimentalmente que quando ha augmento de certos lipoides no sangue (cholesterina), o S. R. E., especialmente o do baço, prolifera intensamente. Nas molestias em que a taxa dos lipoides se eleva no sangue, os histiocytos esplenicos se carregam de gotticulas lipoidicas (diabete lipemica). O S. R. E. do baço participaria nesse caso da elaboração dos lipoides.

Por outro lado, si se extirpar o baço de animaes tratados pela cholesterina, a taxa desta no sangue augmenta ao em vez de diminuir.

Quanto aos proteicos: uma dieta rica de albuminoides provoca no baço modificações do S. R. E. parecidas com as da lipoidemia.

5) - DEFEZA CELlular, IMMUNidAde, ETc. - A defeza cellular é uma das fundamentaes e foi descoberta por MetchNIKOFF. As cellulas do S. R. E. correspondem aos "macrophagos" daquelle A., nome dado devido a propriedade que têm de phagocytar as maiores particulas extranhas, ao organismo. Correspondem aos "microphagos" de MetschnikofF os leucocytos polymorphonucleares sanguineos.

E' tão sensivel a faculdade de defeza das cellulas do systema frente a corpos estranhos e a germens penetrados no organismo, que ellas reagem quasi sempre de um modo especial diante de cada agente dessa natureza.

Alem disso, o S. R. E. goza da propriedade de intervir na funcção de immunidade, isto é a propriedade que tem o organismo de fabricar̂ certas substancias defensivas contra os agentes microbianos ou seus productos. Mas tudo isso será assumpto de cursos ulteriores.

Outras funcções cabem ainda ao systema, que aqui não tratamos, visto que seu conhecimento exhorbita de nosso ambito.

Não devemos esquecer porém, as funcções do reticulo: segundo a escola italiana, pelo estroma reticulado vehicular- 
se-iam as substancias elaboradas pelo systema quer, ao nivel dos endothelios, quer ao nivel das cellulas reticulares. 0 reticulo dos capillares regulariam ainda, segundo varios A.A., as modificações de calibre da luz desses vasos.

Terminando, fica assim estabelecida uma grande importancia nas funcções do S. R. E., graças ás quaes, o tecido connectivo perdeu o significado de simples tecido de sustentação para entrar na categoria dos chamados tecidos nobres, com o nome de tecido tropho-connectival (RufFinI).

Maio, 1930. 